Pl. Syst. Evol. 184: 77-87 (1993)

Systematics

and

Evolution

C Springer-Verlag 1993

Printed in Austria

\title{
Cytological analysis of complex-heterozygotes in populations of Oenothera grandiflora (Onagraceae) in Alabama
}

\author{
Elfriede Schumacher and Erich Steiner
}

Received March 11, 1992

Key words: Onagraceae, Oenothera grandiflora, O. biennis, O. villosa. - Chromosome arrangements, complex-heterozygotes, incompatibility alleles, populations. - Flora of Alabama.

\begin{abstract}
Genetic analysis of unusual complex-heterozygous genotypes in populations of $O$. grandiflora from Alabama (USA) has shown that these strains are composed of a typical grandiflora (B) complex and an altered $\mathrm{B}$ complex (designated as $\mathrm{B}^{\mathrm{A}}$ ) which probably contains genetic elements derived from an A genotype such as the beta complex of $O$. biennis group 1. Analysis of the meiotic configurations of artificial hybrids between the new strains and a series of complexes of known segmental arrangement allowed determination of the arrangements of the unknown complexes. These data are evidence for origin of the altered $\mathrm{B}$ complexes.
\end{abstract}

Until the late 1970's Oenothera grandiflora was regarded as a self-compatible, structurally homozygous relic species (CLELAND 1972), the members of which more or less uniformly possessed the ancestral segmental arrangement of chromosomes characteristic of subsect. Oenothera, namely $1 \cdot 2 \quad 3.4 \quad 5.6 \quad 7 \cdot 10 \quad 9.8 \quad 11 \cdot 12 \quad 13 \cdot 14$ (STEINER 1952). The discovery of self-incompatible individuals in several populations in Alabama (STUBbe \& RAven 1979) stimulated interest in a more detailed study of this species which is believed to be one of the progenitors of the complexheterozygous species, $O$. biennis and $O$. parviflora. $O$. grandiflora is distributed throughout the southeastern United States and is not restricted to the vicinity of Mobile, Alabama, as once thought. Study of earlier, as well as more recent collections from a number of different localities in Alabama (STEINER \& STUBBe 1984, 1986) brought new insights into the population structure of $O$. grandiflora; a greater diversity of chromosome arrangements was observed and a number of structurally heterozygous individuals was found, as to be expected for an open-pollinated species, their occurrence apparently related to the presence of numerous Si alleles.

Special attention was directed toward several unusual genotypes within populations of $O$. grandiflora which were complex-heterozygotes with a meiotic config- 
Table 1. Representative strains from Oenothera grandiflora populations in Alabama analysed in the current study. For geographic origin see SteINER \& STUbBe (1986)

\begin{tabular}{lll}
\hline Strain & $\begin{array}{l}\text { Chromosome } \\
\text { configuration }\end{array}$ & $\begin{array}{l}\text { Original culture } \\
\text { number }\end{array}$ \\
\hline Bay Minette A-6 & 7 pairs & $84 / 340-1$ \\
Bigbee 2 & 7 pairs & $84 / 372-1$ \\
Bolinger 4 & 7 pairs & $84 / 363-1$ \\
Cantonment 1 & 7 pairs & $84 / 331-3$ \\
Castleberry A-1/1 & $\odot 10,2$ pairs & $84 / 301-1$ \\
Castleberry A-1/2 & $\odot 10,2$ pairs & $84 / 301-2$ \\
Castleberry A-4 & $\odot 12,1$ pair & $84 / 304-1$ \\
Castleberry A-7 & $\odot 14$ & $84 / 307-1$ \\
Castleberry A-9 & $\odot 10,2$ pairs & $84 / 309-1$ \\
Castleberry B-8 & 7 pairs & $84 / 318-7$ \\
Chastang 1 & $\odot 12,1$ pair & $84 / 350-3$ \\
Chastang 7 & $\odot 8,3$ pairs & $84 / 356-1$ \\
County Road 6-1 & $\odot 4,5$ pairs & $84 / 368-1$ \\
Flomaton 1 & 7 pairs & $84 / 321-1$ \\
Flomaton 6 & $\odot 4, \odot 4,3$ pairs & $84 / 326-1$ \\
Frankville & 7 pairs & $84 / 370-2$ \\
Sims Chapel & 7 pairs & $84 / 359 \mathrm{~b}$ \\
\hline
\end{tabular}

uration of either a circle of 10 and 2 pairs, a circle of 12 and 1 pair, or a circle of 14 chromosomes. Genetic analysis of the complex-heterozygotes has shown them to be composed of a typical grandiflora complex (B type) and a complex which is distinctly different, tending phenotypically toward the A type characteristic of $O$. villosa and the beta complexes of the $O$. biennis group 1 . This latter complex, designated as $\mathrm{B}^{\mathrm{A}}$, generally lacks an $\mathrm{Si}$ allele or a lethal; therefore, upon selfing of the complex-heterozygotes viable homozygotes, $\mathrm{B}^{\mathrm{A}} \mathrm{B}^{\mathrm{A}}$, may appear among the progeny, although in varying numbers, depending upon the particular strain.

The origin of the $\mathrm{B}^{\mathrm{A}}$ genotype is of particular interest. To test the hypothesis that hybridization between $O$. grandiflora and $O$. biennis played a role in the evolution of this new genotype, it is necessary to determine the segmental arrangement of its chromosomes and to compare it with those of the homozygous strains of $O$. grandiflora and of $O$. biennis. This is a report of such cytological analysis.

\section{Material and methods}

The strains under investigation were obtained from seeds collected in Alabama as reported in Steiner \& Stubbe (1986). They are listed in Table 1 together with their meiotic configurations and initial culture numbers. The complexes of these strains were combined with a series of complexes of known segmental arrangement (Table 2). Analysis of the meiotic 
Table 2. Chromosome formulae of complexes used in the present analysis

\begin{tabular}{|c|c|c|c|c|c|c|c|}
\hline \multirow{2}{*}{$\frac{\text { Complex }}{\text { hhookeri deVries }}$} & \multicolumn{7}{|c|}{ Segmental arrangement } \\
\hline & $1 \cdot 2$ & $3 \cdot 4$ & $5 \cdot 6$ & $7 \cdot 8$ & $9 \cdot 10$ & $11 \cdot 12$ & $13 \cdot 14$ \\
\hline hookeri Johansen & $1 \cdot 2$ & $3 \cdot 4$ & $5 \cdot 6$ & $7 \cdot 10$ & $9 \cdot 8$ & $11 \cdot 12$ & $13 \cdot 14$ \\
\hline helata Guatemala & $1 \cdot 2$ & $3 \cdot 10$ & $5 \cdot 9$ & $7 \cdot 4$ & $6 \cdot 8$ & $11 \cdot 12$ & $13 \cdot 14$ \\
\hline belata Cholula & $1 \cdot 4$ & $3 \cdot 2$ & $5 \cdot 9$ & $7 \cdot 10$ & $6 \cdot 8$ & $11 \cdot 12$ & $13 \cdot 14$ \\
\hline${ }^{\mathrm{h}}$ grandiflora Brewton & $1 \cdot 3$ & $2 \cdot 4$ & $5 \cdot 14$ & $7 \cdot 10$ & $9 \cdot 8$ & $11 \cdot 12$ & $13 \cdot 6$ \\
\hline${ }_{\text {grandiflora York }}$ & $1 \cdot 2$ & $3 \cdot 4$ & $5 \cdot 6$ & $7 \cdot 10$ & $9 \cdot 8$ & $11 \cdot 12$ & $13 \cdot 14$ \\
\hline excellens chicaginensis & $1 \cdot 2$ & $3 \cdot 4$ & $5 \cdot 6$ & $7 \cdot 10$ & $9 \cdot 8$ & $11 \cdot 12$ & $13 \cdot 14$ \\
\hline punctulans chicaginensis & $1 \cdot 4$ & $3 \cdot 9$ & $5 \cdot 2$ & $7 \cdot 8$ & $6 \cdot 12$ & $11 \cdot 10$ & $13 \cdot 14$ \\
\hline undans hungarica & $1 \cdot 4$ & $3 \cdot 2$ & $5 \cdot 10$ & $7 \cdot 6$ & $9 \cdot 13$ & $11 \cdot 12$ & $8 \cdot 14$ \\
\hline$\alpha$ Forsberg & $1 \cdot 7$ & $3 \cdot 4$ & $5 \cdot 8$ & $2 \cdot 10$ & $9 \cdot 11$ & $6 \cdot 12$ & $13 \cdot 14$ \\
\hline$\alpha$ Fruitland & $1 \cdot 2$ & $3 \cdot 5$ & $6 \cdot 12$ & $7 \cdot 10$ & $9 \cdot 4$ & $11 \cdot 8$ & $13 \cdot 14$ \\
\hline$\alpha$ lowa II & $1 \cdot 2$ & $3 \cdot 14$ & $5 \cdot 6$ & $7 \cdot 11$ & $9 \cdot 6$ & $10 \cdot 12$ & $13 \cdot 4$ \\
\hline$\alpha$ Littleton & $1 \cdot 10$ & $3 \cdot 4$ & $5 \cdot 8$ & $7 \cdot 2$ & $9 \cdot 11$ & $6 \cdot 12$ & $13 \cdot 14$ \\
\hline$\alpha$ Monett & $1 \cdot 2$ & $3 \cdot 10$ & $5 \cdot 12$ & $7 \cdot 14$ & $9 \cdot 8$ & $11 \cdot 6$ & $13 \cdot 4$ \\
\hline$\alpha$ Yamika & $1 \cdot 8$ & $3 \cdot 14$ & $5 \cdot 11$ & $7 \cdot 10$ & $9 \cdot 12$ & $2 \cdot 6$ & $13 \cdot 4$ \\
\hline gaudens lamarckiana & $1 \cdot 2$ & $3 \cdot 12$ & $5 \cdot 6$ & $7 \cdot 11$ & $9 \cdot 4$ & $8 \cdot 14$ & $13 \cdot 10$ \\
\hline velans lamarckiana & $1 \cdot 2$ & $3 \cdot 4$ & $5 \cdot 8$ & $7 \cdot 6$ & $9 \cdot 10$ & $11 \cdot 12$ & $13 \cdot 14$ \\
\hline blandina & $1 \cdot 2$ & $3 \cdot 4$ & $5 \cdot 6$ & $7 \cdot 10$ & $9 \cdot 14$ & $11 \cdot 12$ & $13 \cdot 8$ \\
\hline$\alpha$ Bestwater & $1 \cdot 4$ & $3 \cdot 8$ & $5 \cdot 14$ & $7 \cdot 10$ & $9 \cdot 2$ & $11 \cdot 12$ & $13 \cdot 6$ \\
\hline$\beta$ Bestwater & $1 \cdot 14$ & $3 \cdot 2$ & $5 \cdot 9$ & $7 \cdot 8$ & $6 \cdot 12$ & $11 \cdot 10$ & $13 \cdot 4$ \\
\hline$\alpha$ Birch Tree II & $1 \cdot 2$ & $3 \cdot 4$ & $5 \cdot 14$ & $7 \cdot 10$ & $9 \cdot 8$ & $11 \cdot 12$ & $13 \cdot 6$ \\
\hline$\beta$ Birch Tree II & $1 \cdot 13$ & $3 \cdot 2$ & $5 \cdot 7$ & $4 \cdot 12$ & $9 \cdot 6$ & $11 \cdot 10$ & $8 \cdot 14$ \\
\hline$\alpha$ Camp Peary & $1 \cdot 2$ & $3 \cdot 4$ & $5 \cdot 14$ & $7 \cdot 10$ & $9 \cdot 8$ & $11 \cdot 12$ & $13 \cdot 6$ \\
\hline$\alpha$ Delaware & $1 \cdot 2$ & $3 \cdot 4$ & $5 \cdot 14$ & $7 \cdot 10$ & $9 \cdot 8$ & $11 \cdot 12$ & $13 \cdot 6$ \\
\hline$\beta$ Delaware & $1 \cdot 4$ & $3 \cdot 8$ & $5 \cdot 7$ & $2 \cdot 14$ & $9 \cdot 6$ & $11 \cdot 10$ & $13 \cdot 12$ \\
\hline$\alpha$ Lanoraie & $1 \cdot 4$ & $3 \cdot 12$ & $5 \cdot 2$ & $7 \cdot 11$ & $9 \cdot 8$ & $6 \cdot 10$ & $13 \cdot 14$ \\
\hline$\beta$ Lanoraie & $1 \cdot 2$ & $3 \cdot 12$ & $5 \cdot 6$ & $7 \cdot 4$ & $9 \cdot 11$ & $10 \cdot 14$ & $13 \cdot 8$ \\
\hline$\alpha$ Richmond & $1 \cdot 9$ & $3 \cdot 10$ & $5 \cdot 14$ & $7 \cdot 4$ & $2 \cdot 8$ & $11 \cdot 12$ & $13 \cdot 6$ \\
\hline albicans suaveolens Grado & $1 \cdot 12$ & $3 \cdot 6$ & $5 \cdot 7$ & $2 \cdot 14$ & $9 \cdot 4$ & $11 \cdot 10$ & $13 \cdot 8$ \\
\hline flavens suaveolens Grado & $1 \cdot 4$ & $3 \cdot 2$ & $5 \cdot 6$ & $7 \cdot 10$ & $9 \cdot 8$ & $11 \cdot 12$ & $13 \cdot 14$ \\
\hline albicans biennis deVries & $1 \cdot 4$ & $3 \cdot 6$ & $5 \cdot 7$ & $2 \cdot 14$ & $9 \cdot 8$ & $11 \cdot 10$ & $13 \cdot 12$ \\
\hline$\alpha$ Buck Creek & $1 \cdot 4$ & $3 \cdot 10$ & $5 \cdot 7$ & $2 \cdot 6$ & $9 \cdot 8$ & $11 \cdot 12$ & $13 \cdot 14$ \\
\hline$\beta$ Buck Creek & $1 \cdot 2$ & $3 \cdot 5$ & $6 \cdot 8$ & $7 \cdot 12$ & $9 \cdot 13$ & $11 \cdot 4$ & $10 \cdot 14$ \\
\hline$\beta$ Elma II & $1 \cdot 2$ & $3 \cdot 12$ & $5 \cdot 6$ & $7 \cdot 10$ & $9 \cdot 4$ & $11 \cdot 13$ & $8 \cdot 14$ \\
\hline$\alpha$ Indian River & $1 \cdot 4$ & $3 \cdot 2$ & $5 \cdot 7$ & $6 \cdot 10$ & $9 \cdot 8$ & $11 \cdot 12$ & $13 \cdot 14$ \\
\hline$\alpha$ Micaville & $1 \cdot 5$ & $3 \cdot 9$ & $2 \cdot 6$ & $7 \cdot 10$ & $4 \cdot 8$ & $11 \cdot 12$ & $13 \cdot 14$ \\
\hline$\alpha$ Wakefield & $1 \cdot 7$ & $3 \cdot 10$ & $5 \cdot 4$ & $2 \cdot 6$ & $9 \cdot 8$ & $11 \cdot 12$ & $13 \cdot 14$ \\
\hline$\beta$ Wakefield & $1 \cdot 2$ & $3 \cdot 5$ & $6 \cdot 8$ & $7 \cdot 12$ & $9 \cdot 13$ & $11 \cdot 4$ & $10 \cdot 14$ \\
\hline$\alpha$ Williamsville & $1 \cdot 4$ & $3 \cdot 2$ & $5 \cdot 6$ & $7 \cdot 10$ & $9 \cdot 8$ & $11 \cdot 12$ & $13 \cdot 14$ \\
\hline rigens ammophila & $1 \cdot 2$ & $3 \cdot 4$ & $5 \cdot 6$ & $7 \cdot 11$ & $9 \cdot 10$ & $8 \cdot 14$ & $13 \cdot 12$ \\
\hline percurvans ammophila & $1 \cdot 14$ & $3 \cdot 5$ & $6 \cdot 8$ & $7 \cdot 10$ & $9 \cdot 2$ & $11 \cdot 4$ & $13 \cdot 12$ \\
\hline$\alpha$ angustissima & $1 \cdot 13$ & $3 \cdot 4$ & $5 \cdot 8$ & $7 \cdot 14$ & $9 \cdot 2$ & $11 \cdot 12$ & $6 \cdot 10$ \\
\hline$\alpha$ Camas & $1 \cdot 9$ & $3 \cdot 5$ & $4 \cdot 6$ & $7 \cdot 10$ & $2 \cdot 8$ & $11 \cdot 14$ & $13 \cdot 12$ \\
\hline$\alpha$ biennis 1 & $1 \cdot 2$ & $3 \cdot 4$ & $5 \cdot 14$ & $7 \cdot 10$ & $9 \cdot 8$ & $11 \cdot 12$ & $13 \cdot 6$ \\
\hline rubens biennis deVries & $1 \cdot 2$ & $3 \cdot 12$ & $5 \cdot 6$ & $7 \cdot 11$ & $9 \cdot 4$ & $8 \cdot 14$ & $13 \cdot 10$ \\
\hline B type Brewton & $1 \cdot 2$ & $3 \cdot 4$ & $5 \cdot 6$ & $7 \cdot 10$ & $9 \cdot 8$ & $11 \cdot 12$ & $13 \cdot 14$ \\
\hline $\mathrm{B}^{\mathrm{A}}$ type Brewton & $1 \cdot 6$ & $3 \cdot 2$ & $5 \cdot 11$ & $7 \cdot 10$ & $9 \cdot 4$ & $8 \cdot 12$ & $13 \cdot 14$ \\
\hline
\end{tabular}


Table 3. Chromosome configurations of hybrids between various homozygous strains of Oenothera grandiflora

\begin{tabular}{ll}
\hline Cross & $\begin{array}{l}\text { Chromosome } \\
\text { configuration }\end{array}$ \\
\hline Bay Minette A-6 $\times$ York & 7 pairs \\
Flomaton $6 \times$ York & 7 pairs \\
York $\times$ Bigbee 2 & $\odot 4,5$ pairs \\
York $\times$ Frankville & $\odot 4, \odot 4,3$ pairs \\
Bigbee $2 \times$ Bolinger 4 & $\odot 4,5$ pairs \\
Castleberry B-8 8 Bolinger 4 & 7 pairs \\
Castleberry B-8 $\times$ Frankville & $\odot 4,5$ pairs \\
Flomaton $1 \times$ Bigbee 2 & 7 pairs \\
Flomaton $1 \times$ Bolinger 4 & $\odot 4,5$ pairs \\
Flomaton $1 \times$ Frankville & 7 pairs \\
County Road 6-1 $\times$ Brewton & 7 pairs \\
Flomaton $1 \times$ County Road 6-1 & $\odot 4,5$ pairs \\
Castleberry B-8 8 Brewton & $\odot 4, \odot 4,3$ pairs \\
Bigbee $2 \times$ Brewton & $\odot 4,5$ pairs \\
Flomaton $1 \times$ Brewton & $\odot 4,5$ pairs \\
Bolinger $4 \times$ York & 7 pairs \\
\hline
\end{tabular}

configurations of these hybrids allowed determination of the arrangements of the unknown complexes.

The large-circled complex-heterozygous strains were first crossed as pollen parents with the artificial heterogamous hybrids albicans.percurvans and albicans'undans as seed parents.

The offspring consisted of twin hybrids in which the egg complex, albicans, was combined with either the typical $B$ complex or with the $B^{A}$ complex. These two types were then combined with a series of complexes of known segmental arrangement. By determining the configuration at meiosis of each of these complex combinations data were obtained that allowed determination of the segmental arrangements of the $B$ and $B^{A}$ complexes of the various collections.

The meiotic chromosome configurations of the hybrids were determined using the acetocarmine method for anther squashes as described by STEINER \& STUbBe (1984). The method of analysis for determining the segmental arrangement of a complex is described by CLELAND $(1972: 61,65)$. Such analysis is now facilitated by a computer program devised by WASMUND (1980).

In addition, appropriate complex combinations permitted recognition of the presence of a self-incompatibility allele in the complexes under investigation.

\section{Results}

Tables 3, 4, 6, and 7 list the chromosome configurations of the new races in combination with those of known segmental arrangements. 
Table 4. Chromosome configurations of hybrids between the type B grandiflora complex of the complex-heterozygous strains and complexes with known segmental arrangements

\begin{tabular}{|c|c|}
\hline Complex combination & $\begin{array}{l}\text { Chromosome } \\
\text { configuration }\end{array}$ \\
\hline albicans Grado $\cdot$ B Castleberry A-1/1 & $\odot 14$ \\
\hline$\beta$ Bestwater $\cdot$ B Castleberry A-1/1 & $\odot 8, \odot 6$ \\
\hline$\alpha$ biennis $1 \cdot \mathrm{B}$ Castleberry A- $1 / 1$ & $\odot 6, \odot 4,2$ pairs \\
\hline hblandina $\cdot$ B Castleberry A-1/1 & $\odot 6, \odot 4,2$ pairs \\
\hline$\beta$ Buck Creek $\cdot$ B Castleberry A-1/1 & $\odot 10, \odot 4$ \\
\hline hookeri deVries $\cdot$ B Castleberry A-1/1 & $\odot 4, \odot 4, \odot 4,1$ pair \\
\hline$\alpha$ Indian River $\cdot \mathrm{B}$ Castleberry A-1/1 & $\odot 4, \odot 4,3$ pairs \\
\hline hhookeri Johansen - B Castleberry A-1/1 & $\odot 4, \odot 4,3$ pairs \\
\hline$\alpha$ Micaville $\cdot$ B Castleberry A- $1 / 1$ & $\odot 8, \odot 4,1$ pair \\
\hline$\alpha$ Williamsville $\cdot$ B Castleberry A-1/1 & $\odot 4,5$ pairs \\
\hline$\alpha$ Delaware $\cdot \mathrm{B}$ Castleberry $\mathrm{A}-1 / 2$ & $\odot 6, \odot 4,2$ pairs \\
\hline${ }^{\mathrm{h}} \mathrm{b}$ landina $\cdot \mathrm{B}$ Castleberry $\mathrm{A}-1 / 2$ & $\odot 6, \odot 4,2$ pairs \\
\hline excellens chicag. $\cdot$ B Castleberry A-1/2 & $\odot 4, \odot 4,3$ pairs \\
\hline hookeri Johansen Castleberry A-1/2 & $\odot 4, \odot 4,3$ pairs \\
\hline albicans Grado $\cdot$ B Castleberry A-4 & $\odot 10, \odot 4$ \\
\hline${ }^{h}$ hookeri deVries $\cdot$ B Castleberry A-4 & $\odot 4, \odot 4,3$ pairs \\
\hline hhookeri Johansen Castleberry A-4 & $\odot 4,5$ pairs \\
\hline$\alpha$ Richmond $\cdot$ B Castleberry A-4 & $\odot 6, \odot 4, \odot 4$ \\
\hline albicans Grado B Castleberry A-7 & $\odot 14$ \\
\hline$\alpha$ Birch Tree II $\cdot$ B Castleberry A-7 & $\odot 4,5$ pairs \\
\hline$\beta$ Birch Tree II - B Castleberry A-7 & $\odot 14$ \\
\hline$\beta$ Buck Creek $\cdot$ B Castleberry A-7 & $\odot 6, \odot 4,2$ pairs \\
\hline elata Cholula $\cdot$ B Castleberry A-7 & $\odot 10,2$ pairs \\
\hline hookeri deVries $\cdot$ B Castleberry A-7 & $\odot 6, \odot 4,2$ pairs \\
\hline$\alpha$ Indian River $\cdot B$ Castleberry A-7 & $\odot 10,2$ pairs \\
\hline${ }^{\text {h}}$ hookeri Johansen $\cdot$ B Castleberry A-7 & $\odot 6,4$ pairs \\
\hline$\alpha$ Lanoraie $\cdot \mathrm{B}$ Castleberry A-7 & $\odot 12,1$ pair \\
\hline$\alpha$ Littleton $\cdot$ B Castleberry A-7 & $\odot 10, \odot 4$ \\
\hline$\alpha$ Micaville $\cdot B$ Castleberry A-7 & $\odot 10,2$ pairs \\
\hline$\alpha$ Wakefield $\cdot$ B Castleberry A-7 & $\odot 10,2$ pairs \\
\hline$\alpha$ Williamsville $\cdot$ B Castleberry A-7 & $\odot 8,3$ pairs \\
\hline${ }^{\text {h}}$ hookeri Johansen $\cdot$ B Castleberry A-9 & 7 pairs \\
\hline albicans Grado $\cdot$ B Chastang 1 & $\odot 14$ \\
\hline$\alpha$ Delaware $\cdot \mathrm{B}$ Chastang 1 & $\odot 4,5$ pairs \\
\hline blandina $\cdot \mathrm{B}$ Chastang 1 & $\odot 8,3$ pairs \\
\hline elata Guatemala $\cdot$ B Chastang 1 & $\odot 10,2$ pairs \\
\hline hookeri deVries · B Chastang 1 & $\odot 6, \odot 4,2$ pairs \\
\hline hhookeri Johansen $\cdot$ B Chastang 1 & $\odot 6,4$ pairs \\
\hline punctulans chicag. B Chastang 1 & $\odot 14$ \\
\hline albicans Grado $\cdot$ B Chastang 7 & $\odot 14$ \\
\hline$\alpha$ biennis $1 \cdot B$ Chastang 7 & $\odot 4, \odot 4,3$ pairs \\
\hline blandina $\cdot$ B Chastang 7 & $\odot 4, \odot 4,3$ pairs \\
\hline hookeri Johansen $\cdot$ B Chastang 7 & $\odot 4,5$ pairs \\
\hline punctulans chicag. $\cdot$ B Chastang 7 & $\odot 10,2$ pairs \\
\hline
\end{tabular}


Table 5. Chromosome formulae of the B complexes of the complex-heterozygous strains newly found in populations of Oenothera grandiflora

\begin{tabular}{llllllll}
\hline Castleberry A-1/1 & $1 \cdot 4$ & $3 \cdot 2$ & $5 \cdot 6$ & $7 \cdot 10$ & $9 \cdot 8$ & $11 \cdot 13$ & $12 \cdot 14$ \\
Castleberry A-4 & $1 \cdot 2$ & $3 \cdot 4$ & $5 \cdot 6$ & $7 \cdot 10$ & $9 \cdot 8$ & $11 \cdot 13$ & $12 \cdot 14$ \\
Castleberry A-7 & $1 \cdot 2$ & $3 \cdot 5$ & $4 \cdot 14$ & $7 \cdot 10$ & $9 \cdot 8$ & $11 \cdot 12$ & $13 \cdot 6$ \\
Castleberry A-9 & $1 \cdot 2$ & $3 \cdot 4$ & $5 \cdot 6$ & $7 \cdot 10$ & $9 \cdot 8$ & $11 \cdot 12$ & $13 \cdot 14$ \\
Chastang 1 & $1 \cdot 2$ & $3 \cdot 5$ & $4 \cdot 14$ & $7 \cdot 10$ & $9 \cdot 8$ & $11 \cdot 12$ & $13 \cdot 6$ \\
Chastang 7 & $1 \cdot 4$ & $3 \cdot 2$ & $5 \cdot 6$ & $7 \cdot 10$ & $9 \cdot 8$ & $11 \cdot 12$ & $13 \cdot 14$ \\
\hline
\end{tabular}

The chromosome formulae of the homozygous strains of $O$. grandiflora determined from these data can be summarized as follows: The strains Bay Minette A6, Bolinger 4, Castleberry B-8 and one of the complexes of Flomaton 6 exhibit the segmental arrangement believed to be ancestral for subsect. Oenothera, namely $1 \cdot 2$ $3.45 .67 \cdot 109.811 \cdot 1213 \cdot 14$. The formulae of the strains Bigbee 2, Flomaton 1, and Frankville are one translocation removed from the foregoing, i.e., $1 \cdot 32 \cdot 45 \cdot 6$ $7 \cdot 109 \cdot 811 \cdot 1213 \cdot 14$. One complex of the strain County Road IV-1 has the same formula as one of the homozygous Brewton races, namely $1 \cdot 32 \cdot 45 \cdot 147 \cdot 109 \cdot 8$ $11 \cdot 1213 \cdot 6$. Cytological data were insufficient to fully establish the segmental arrangements of Cantonment and Sims Chapel, but they appear to be close to the former.

Table 4 presents the meiotic configurations of the hybrids with the $B$ type grandiflora complex found in the complex-heterozygotes of $O$. grandiflora populations. The formulae obtained from the analysis of these data are found in Table 5. Castleberry A-9 carries the ancestral arrangement. Chastang 7 is one interchange removed from the latter, having the 1.4 and 3.2 . Castleberry A-4 is also one interchange removed from the ancestral arrangement, but differs in having the $11 \cdot 13$ and $12 \cdot 14$. Castleberry A-1/1 has a B complex with both interchanges. The translocation $11 \cdot 13$ and $12 \cdot 14$ appears to be rare; it has not previously been found in $O$. grandiflora and has only been reported in certain beta villosa complexes. Castleberry A-7 and Chastang 1, which are identical in segmental arrangement, are two interchanges removed from the ancestral arrangement, but differ by only one interchange from the arrangement most common among the alpha biennis group 1 complexes (designated as arrangement C by STEINER \& STUbBe 1984).

Table 6 lists the meiotic configurations of a series of hybrids combining complexes of known arrangements with the $\mathrm{B}^{\mathrm{A}}$ type complexes found in the complexheterozygotes of the $O$. grandiflora populations. From these data the segmental arrangements of the $\mathrm{B}^{\mathrm{A}}$ complexes were determined. The $\mathrm{B}^{\mathrm{A}}$ complexes of Castleberry $A-1 / 1$ and Chastang 7 proved to be identical with each other as well as with the $\mathrm{B}^{\mathrm{A}}$ complex found in the Brewton population, namely $1.63 \cdot 25 \cdot 117 \cdot 109 \cdot 4$ $8 \cdot 1213 \cdot 14$ (STEINER \& STUBBE 1986). The formula of the $B^{A}$ complex of Castleberry A-7 differs from the foregoing by one interchange involving the most conserved chromosomes $7 \cdot 10$ and $13 \cdot 14$; it is $1.63 \cdot 25 \cdot 117 \cdot 149 \cdot 4 \quad 8 \cdot 1213 \cdot 10$. 
Table 6. Chromosome configurations of hybrids between the $\mathrm{B}^{\mathrm{A}}$ complexes of the complex-heterozygous strains and complexes with known segmental arrangements

Complex combination
Chromosome

configuration

albicans Grado $\cdot \mathrm{B}^{\mathrm{A}}$ Castleberry A-1/1

a Bestwater $\cdot \mathrm{B}^{\mathrm{A}}$ Castleberry $\mathrm{A}-1 / 1$

$\alpha$ biennis $1 \cdot \mathrm{B}^{\mathrm{A}}$ Castleberry $\mathrm{A}-1 / 1$

rubens $\cdot B^{A}$ Castleberry $A-1 / 1$

${ }^{\mathrm{h}}$ blandina $\cdot \mathrm{B}^{\mathrm{A}}$ Castleberry A-1/1

$\alpha$ Buck Creek $\cdot B^{A}$ Castleberry A-1/1

$\alpha$ Delaware $\cdot \mathrm{B}^{\mathrm{A}}$ Castleberry $\mathrm{A}-1 / 1$

$\beta$ Delaware $\cdot B^{A}$ Castleberry A-1/1

$\alpha$ Fruitland $\cdot B^{A}$ Castleberry A-1/1

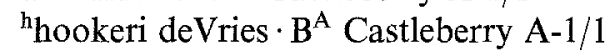

${ }^{h}$ hookeri Johansen $\cdot B^{A}$ Castleberry A-1/1

$\alpha$ Indian River $\cdot B^{A}$ Castleberry A-1/1

$\alpha$ Lanoraie $\cdot \mathrm{B}^{\mathrm{A}}$ Castleberry A-1/1

$\beta$ Lanoraie $\cdot B^{A}$ Castleberry A-1/1

$\alpha$ Littleton $\cdot \mathrm{B}^{\mathrm{A}}$ Castleberry A-1/1

$\alpha$ Monett $\cdot \mathrm{B}^{\mathrm{A}}$ Castleberry $\mathrm{A}-1 / 1$

$a$ Richmond - $\mathrm{B}^{\mathrm{A}}$ Castleberry A-1/1

velans lamarckiana $\cdot B^{A}$ Castleberry A-1/1

$\beta$ Wakefield $\cdot B^{A}$ Castleberry A-1/1

$\alpha$ Yakima $\cdot \mathrm{B}^{\mathrm{A}}$ Castleberry A-1/1

albicans Grado $\cdot B^{A}$ Castleberry A-1/2

$\alpha$ Bestwater $\cdot \mathrm{B}^{\mathrm{A}}$ Castleberry A-1/2

$\alpha$ biennis $1 \cdot \mathrm{B}^{\mathrm{A}}$ Castleberry A-1/2

${ }^{\mathrm{b}}$ blandina $\cdot \mathrm{B}^{\mathrm{A}}$ Castleberry A-1/2

$\alpha$ Camas $\cdot B^{A}$ Castleberry A-1/2

$\alpha$ Forsberg $\cdot \mathrm{B}^{\mathrm{A}}$ Castleberry $\mathrm{A}-1 / 2$

${ }^{\mathrm{h}}$ hookeri deVries $\cdot \mathrm{B}^{\mathrm{A}}$ Castleberry A-1/2

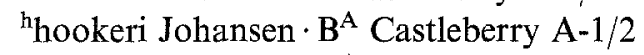

punctulans chicag. $B^{A}$ Castleberry A-1/2

gaudens lamarckiana $\cdot B^{A}$ Castleberry A-4

hhookeri deVries $\cdot B^{A}$ Castleberry A-4

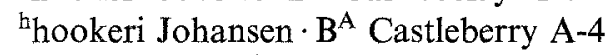

$\alpha$ Richmond $\cdot \mathrm{B}^{\mathrm{A}}$ Castleberry A-4

velans lamarckiana $\cdot B^{A}$ Castleberry A-4

albicans Grado $\cdot B^{A}$ Castleberry A-7

a Bestwater $\cdot \mathrm{B}^{\mathrm{A}}$ Castleberry A-7

$\alpha$ Buck Creek $\cdot B^{A}$ Castleberry A-7

$\alpha$ Camp Peary $\cdot B^{A}$ Castleberry A-7

elata Cholula $\cdot B^{A}$ Castleberry A-7

gaudens lamarckiana $\cdot B^{A}$ Castleberry $A-7$

hookeri deVries $\cdot \mathrm{B}^{\mathrm{A}}$ Castleberry A-7

hookeri Johansen $\cdot B^{A}$ Castleberry A-7

$\alpha$ Indian River $\cdot \mathrm{B}^{\mathrm{A}}$ Castleberry A-7 $\odot 8, \odot 4,1$ pair

$\odot 12,1$ pair

$\odot 12,1$ pair

$\odot 12,1$ pair

$\odot 12,1$ pair

$\odot 12,1$ pair

$\odot 12,1$ pair

$\odot 6, \odot 4, \odot 4$

$\odot 8,3$ pairs

$\odot 12,1$ pair

$\odot 10,2$ pairs

$\odot 10,2$ pairs

$\odot 12,1$ pair

○ 14

$\odot 12,1$ pair

$\odot 14$

$\odot 14$

$\odot$ 8, $\odot$ 4, 1 pair

$\odot 14$

10, 2 pairs

$\odot$ 8, $\odot$ 4, 1 pair

$\odot 12,1$ pair

$\odot 12,1$ pair

$\odot$ 12, 1 pair

$\odot$ 8, $\odot$ 4, 1 pair

$\odot 12,1$ pair

$\odot$ 12, 1 pair

$\odot 10,2$ pairs

(12, 1 pair

12, 1 pair

$\odot 12,1$ pair

$\bigcirc 10,2$ pairs

○ 14

$\odot$ 8, $\odot$ 4, 1 pair

$\odot 12,1$ pair

$\odot 14$

$\odot 14$

$\odot 14$

$\odot 8, \odot 4,1$ pair

$\odot 10,2$ pairs

$\odot 14$

$\odot 10, \odot 4$

$\odot$ 12, 1 pair 
Table 6 (continued)

\begin{tabular}{|c|c|}
\hline Complex combination & $\begin{array}{l}\text { Chromosome } \\
\text { configuration }\end{array}$ \\
\hline$\alpha$ Littleton $\cdot \mathrm{B}^{\mathrm{A}}$ Castleberry A-7 & $\odot 14$ \\
\hline$\beta$ Wakefield $\cdot B^{A}$ Castleberry A-7 & $\odot 14$ \\
\hline$\alpha$ Williamsville $\cdot \mathrm{B}^{\mathrm{A}}$ Castleberry A-7 & $\odot 8, \odot 4,1$ pair \\
\hline$\alpha$ angustissima $\cdot \mathrm{B}^{\mathrm{A}}$ Chastang 1 & $\odot 6, \odot 4, \odot 4$ \\
\hline$\alpha$ biennis $1 \cdot \mathrm{B}^{\mathrm{A}}$ Chastang 1 & $\odot 12,1$ pair \\
\hline${ }^{\mathrm{h}}$ blandina $\cdot \mathrm{B}^{\mathrm{A}}$ Chastang 1 & $\odot 12,1$ pair \\
\hline$\beta$ Elma II $\cdot \mathrm{B}^{\mathrm{A}}$ Chastang 1 & $\odot 10,2$ pairs \\
\hline$\alpha$ Forsberg $\cdot \mathrm{B}^{\mathrm{A}}$ Chastang 1 & $\odot 12,1$ pair \\
\hline hhookeri deVries $\cdot \mathrm{B}^{\mathrm{A}}$ Chastang 1 & $\odot 12,1$ pair \\
\hline hookeri Johansen $\cdot \mathrm{B}^{\mathrm{A}}$ Chastang 1 & $\odot 10,2$ pairs \\
\hline punctulans chicag. $\cdot \mathrm{B}^{\mathrm{A}}$ Chastang 1 & $\odot 12,1$ pair \\
\hline albicans Grado $\cdot B^{A}$ Chastang 7 & $\odot 8, \odot 4,1$ pair \\
\hline rubens $\cdot \mathrm{B}^{\mathrm{A}}$ Chastang 7 & $\odot 12,1$ pair \\
\hline${ }^{\mathrm{b}}$ blandina $\cdot \mathrm{B}^{\mathrm{A}}$ Chastang 7 & $\odot 12,1$ pair \\
\hline$\alpha$ Birch tree II $\cdot \mathrm{B}^{\mathrm{A}}$ Chastang 7 & $\odot 12,1$ pair \\
\hline$\alpha$ Fruitland $\cdot B^{A}$ Chastang 7 & $\odot 8,3$ pairs \\
\hline${ }^{\mathrm{h}}$ hookeri deVries $\cdot \mathrm{B}^{\mathrm{A}}$ Chastang 7 & $\odot 12,1$ pair \\
\hline hookeri Johansen $\cdot \mathrm{B}^{\mathrm{A}}$ Chastang 7 & $\odot 10,2$ pairs \\
\hline$\alpha$ Indian River $\cdot \mathrm{B}^{\mathrm{A}}$ Chastang 7 & $\odot 10,2$ pairs \\
\hline$\alpha$ Iowa II $\cdot \mathrm{B}^{\mathrm{A}}$ Chastang 7 & $\odot 8, \odot 6$ \\
\hline$\alpha$ Monett $\cdot \mathrm{B}^{\mathrm{A}}$ Chastang 7 & $\odot 14$ \\
\hline$\alpha$ Richmond $\cdot B^{A}$ Chastang 7 & $\odot 14$ \\
\hline rigens ammophila $\cdot \mathrm{B}^{\mathrm{A}}$ Chastang 7 & $\odot 10, \odot 4$ \\
\hline$\alpha$ Yakima $\cdot \mathrm{B}^{\mathrm{A}}$ Chastang 7 & $\odot 10,2$ pairs \\
\hline$\alpha$ Williamston $\cdot \mathrm{B}^{\mathrm{A}}$ Chastang 7 & $\odot 12,1$ pair \\
\hline$\alpha$ Lanoraie $\cdot \mathrm{B}^{\mathrm{A}}$ Chastang 7 & $\odot 12,1$ pair \\
\hline
\end{tabular}

Table 7. Chromosome configurations of hybrids between $\mathrm{B}^{\mathrm{A}}$ complexes confirming the identity of their chromosome formulae

\begin{tabular}{ll}
\hline Cross & $\begin{array}{l}\text { Chromosome } \\
\text { configuration }\end{array}$ \\
\hline Brewton $\times$ Castleberry A-1/1 & 7 pairs \\
Chastang $7 \times$ Brewton & 7 pairs \\
Brewton $\times$ Castleberry A-4 & 7 pairs \\
Castleberry A-1 $1 \times$ Castleberry A-4 & 7 pairs \\
Castleberry A-9 $\times$ Castleberry A-4 & 7 pairs \\
Castleberry A-1 $/ 2 \times$ Castleberry A-4 & 7 pairs \\
Castleberry A-1 $2 \times$ Castleberry A-9 & 7 pairs \\
\hline
\end{tabular}


Table 8. Chromosomal end arrangements found among Oenothera grandiflora strains from Alabama, Florida, and Tennessee

\begin{tabular}{|c|c|c|c|c|c|c|c|}
\hline \multicolumn{7}{|c|}{ End arrangement } & \multirow{2}{*}{$\frac{\text { Designation }}{\mathrm{A}}$} \\
\hline $1 \cdot 2$ & $3 \cdot 4$ & $5 \cdot 6$ & $7 \cdot 10$ & $9 \cdot 8$ & $11 \cdot 12$ & $13 \cdot 14$ & \\
\hline $1 \cdot 4$ & $3 \cdot 2$ & $5 \cdot 6$ & $7 \cdot 10$ & $9 \cdot 8$ & $11 \cdot 12$ & $13 \cdot 14$ & B \\
\hline $1 \cdot 2$ & $3 \cdot 4$ & $5 \cdot 14$ & $7 \cdot 10$ & $9 \cdot 8$ & $11 \cdot 12$ & $13 \cdot 6$ & $\mathrm{C}$ \\
\hline $1 \cdot 2$ & $3 \cdot 4$ & $5 \cdot 13$ & $7 \cdot 10$ & $9 \cdot 8$ & $11 \cdot 12$ & $6 \cdot 14$ & $\mathrm{D}$ \\
\hline $1 \cdot 4$ & $3 \cdot 2$ & $5 \cdot 14$ & $7 \cdot 10$ & $9 \cdot 8$ & $11 \cdot 12$ & $13 \cdot 6$ & $\mathrm{E}$ \\
\hline $1 \cdot 2$ & $3 \cdot 4$ & $5 \cdot 6$ & $7 \cdot 10$ & $9 \cdot 14$ & $11 \cdot 12$ & $13 \cdot 8$ & $\mathrm{~F}$ \\
\hline $1 \cdot 3$ & $2 \cdot 4$ & $5 \cdot 14$ & $7 \cdot 10$ & $9 \cdot 8$ & $11 \cdot 12$ & $13 \cdot 6$ & $\mathrm{G}$ \\
\hline $1 \cdot 2$ & $3 \cdot 5$ & $4 \cdot 14$ & $7 \cdot 10$ & $9 \cdot 8$ & $11 \cdot 12$ & $13 \cdot 6$ & $\mathrm{H}$ \\
\hline $1 \cdot 4$ & $3 \cdot 2$ & $5 \cdot 6$ & $7 \cdot 10$ & $9 \cdot 8$ & $11 \cdot 13$ & $12 \cdot 14$ & $\mathrm{I}$ \\
\hline $1 \cdot 2$ & $3 \cdot 4$ & $5 \cdot 6$ & $7 \cdot 10$ & $9 \cdot 8$ & $11 \cdot 13$ & $12 \cdot 14$ & $\mathrm{~K}$ \\
\hline $1 \cdot 3$ & $2 \cdot 4$ & $5 \cdot 6$ & $7 \cdot 10$ & $9 \cdot 8$ & $11 \cdot 12$ & $13 \cdot 14$ & $\mathrm{~L}$ \\
\hline
\end{tabular}

Crosses among the $\mathrm{B}^{\mathrm{A}}$ complexes (Table 7) show that the $\mathrm{B}^{\mathrm{A}}$ complexes of Castleberry A-1/1, A-1/2, A-4, A-9, and Chastang 7 are identical in segmental arrangement.

\section{Discussion}

The various segmental arrangements (designated A-E) of typical ${ }^{h}$ grandiflora (B type) complexes previously reported by STEINER \& STUBbe (1984) are here supplemented by six new formulae shown in Table 8 . All eleven formulae have the $7 \cdot 10$ in common, ten of the eleven have the 9.8 and nine of the eleven the 11.12 chromosome. The remaining chromosomes show more variability; the reason for this difference is unknown.

The Castleberry and Chastang strains showing circles of 10,12 or 14 chromosomes at meiosis are clearly composed of two different complexes, one a B type typical of $O$. grandiflora, and the second a B type that has been modified in the direction of the A type found in $O$. villosa as well as in the beta complexes of the biennis group 1 of $O$. biennis. The latter species is sympatric with $O$. grandiflora in Alabama and hybrids between the two have been found (STEINER \& STUBbe 1986). Nevertheless, the large-circled strains that carry a $\mathrm{B}^{\mathrm{A}}$ complex cannot be simple hybrids between $O$. grandiflora and $O$. biennis, as already pointed out by STEINER \& STubBe (1986). It is likely that the $\mathrm{B}^{\mathrm{A}}$ complex has been derived from unstable hybrids between $O$. grandiflora as seed parent and $O$. biennis as pollen parent. The integration of some of the beta biennis 1 chromosomes into a ${ }^{h}$ grandiflora complex could have occurred in such a hybrid. For example, if a hybrid forms a circle of 10 and a circle of 4 and the latter is free of lethals, a ${ }^{h}$ grandiflor $a$ complex carrying two beta biennis 1 chromosomes is a possible segregate. Other mechanisms of integration are also possible, e.g., a translocation between the two complexes of a hybrid after the interlocking of chromosomes. The question remains as to which 
chromosomes, or parts of chromosomes, of the $\mathrm{B}^{\mathrm{A}}$ complex are $\mathrm{B}$ or $\mathrm{A}$ type chromosomes.

A comparison of the most common $\mathrm{B}^{\mathrm{A}}$ arrangement $\left(\begin{array}{lllll}1 \cdot 6 & 3 \cdot 2 & 5 \cdot 11 & 7 \cdot 10 & 9 \cdot 4\end{array}\right.$ $8 \cdot 1213 \cdot 14)$ with the arrangements of the beta complexes of $O$. biennis growing in association with the complex-heterozygotes of $O$. grandiflor $a$ would be instructive; unfortunately at the time of $O$. grandiflora collections were made, a parallel collection of $O$. biennis strains from the same range did not seem likely to yield new information and was not made. Of the few arrangements determined for biennis 1 strains from Alabama earlier (STEINER 1952), most carry the 5.11, 8.12, and 13.14 chromosomes. The 13.14 is regularly present in the $\mathrm{B}$ complexes, but the remaining two may be the A chromosomes (or modifications thereof) of the $\mathrm{B}^{\mathrm{A}}$ arrangement. The 3.2 and 7.10 are most likely B chromosomes. In the complex-heterozygotes with a circle of 10 and 2 pairs the pairs are formed by the $7 \cdot 10$ and $13 \cdot 14$ and are in all probability homozygous. The 1.6 and 9.4 chromosomes cannot be classified because of their absence in closely related or associated strains. They may be of hybrid origin, arising from a translocation between an A and a B complex.

Ultimately the analysis of the gene content of single chromosomes may be necessary in order to confirm the presumed hybrid origin of the newly discovered complex-heterozygotes of $O$. grandiflora.

Whether or not the complex-heterozygotes of $O$. grandiflora will play a role in the further evolution of the species is difficult to say. Presumably the hybridity might confer a selective advantage, thus favoring a gradual spread of the $\mathrm{B}^{\mathrm{A}}$ genome with an ultimate shift to populations which are predominantly complex-heterozygous rather than structurally homozygous. At present they are no true breeding when selfed. Their flower structure favors cross pollination. Nevertheless, the $B^{\AA}$ genome appears to have become established in a number of different local populations in southern Alabama; since it seems unlikely that its distribution can be explained through a multiple origin of the genotype, one is led to conclude that its spread is already in progress.

Financial support for this work was provided in part by a grant from the Deutsche Forschungsgemeinschaft to Prof. Dr WiLfRIEd STUBbE.

\section{References}

Cleland, R. E., 1972: Oenothera: cytogenetics and evolution. - New York: Academic Press.

STEINER, E. E., 1952: Phylogenetic studies in Euoenothera. - Evolution 6: 69-80.

Steiner, E., Stubbe, W., 1984: A contribution to the population biology of Oenothera grandiflora L'HeR. - Amer. J. Bot. 71: 1293-1301.

- - 1986: Oenothera grandiflora revisited: a new view of its population structure. Bull. Torrey Bot. Club 113: 406-412.

Stubbe, W., Raven, P. H., 1979: Genetic self-incompatibility in Oenothera subsect. Euoenothera. - Science 204: 327.

WASMUND, O. W., 1980: Zytogenetische Untersuchungen zur Systematik einiger Sippen der Subsektion Euoenothera der Gattung Oenothera (Onagraceae). - Staatsexamen, Universität Düsseldorf. 
Addresses of the authors: Mrs Elfriede Schumacher, Botanisches Institut, HeinrichHeine-Universität, D-W-4000 Düsseldorf, Federal Republic of Germany. - Dr ERICH STEINER, Department of Biology, University of Michigan, Ann Arbor, Michigan, 48109, USA.

Accepted September 2, 1992 by F. EHRENDOFER 